\title{
Emergência e desenvolvimento inicial de plântulas de amendoim submetidas ao uso de extrato pirolenhoso
}

\author{
Submetido - 04 Jul. 2021 \\ Aprovado - 31 jul. 2021 \\ Publicado - 09 dez. 2021
}

http://dx.doi.org/10.52755/sas.v.2i(edesp1)128

César Martoreli da Silveira iD

Engenheiro Agrônomo, Prof. Dr., Universidade Estadual Paulista - Colégio Técnico Agrícola Faculdade de Ciências Agrárias e Veterinárias - CTA/FCAV - UNESP, Jaboticabal-SP. E-mail: cesar.m.silveira@unesp.br.

Gabriel Vinícius Lima de Souza iD

Graduando Engenharia Agronômica, Universidade Estadual Paulista - Faculdade de Ciências Agrárias e Veterinárias - FCAV/UNESP, Jaboticabal-SP. E-mail: gv.souza@unesp.br.

José Eduardo Fadim Junior (i) Fundação Educacional do Centro Universitário de Barretos - UNIFEB, Barretos-SP. E-mail: juninhofadim95@gmail.com.

Engenheiro Agrônomo, MSc., Consultor e Pesquisador Autônomo, Jaboticabal-SP. E-mail: cr.detoffoli@gmail.com.

\section{RESUMO}

O extrato pirolenhoso (EP), produto proveniente da queima da madeira, conhecido como ácido pirolenhoso ou vinagre de madeira, tem sido estudado em diversas culturas como milho, feijão, arroz, capazes de exercer efeitos na germinação e vigor das sementes. A cultura do amendoim tem importância socioeconômica nas regiões de sua produção e sempre busca inserir novas tecnologias. Neste sentido, o objetivo deste estudo foi avaliar a influência do extrato pirolenhoso na emergência e desenvolvimento inicial de plântulas de amendoim. 0 experimento foi conduzido em delineamento inteiramente casualizado, em esquema fatorial 2x5, com duas cultivares de amendoim: IAC 503 e Granoleico; e cinco doses de EP: T1- 10,0 mL de água deionizada $\mathrm{kg}^{-1}$ de sementes; T2- 2,5 $\mathrm{mL} E P+7,5 \mathrm{~mL}$ de água deionizada $\mathrm{kg}^{-1}$ de sementes; T3- 5,0 mL EP + 5,0 mL de água deionizada $\mathrm{kg}^{-1}$ de sementes; T4- 7,5 mL EP + 2,5 mL de água deionizada $\mathrm{kg}^{-1}$ de sementes e; T5- 10,0 mL EP $\mathrm{kg}^{-1}$ de sementes, com 4 repetições. A semeadura foi realizada em vasos de polietileno de $11 \mathrm{dm}^{3}$, sendo avaliados os parâmetros de porcentagem de emergência (E\%), índice de velocidade de emergência (IVE), comprimento da parta aérea (CPA), da raiz (CPR) e massa de matéria seca de plântula (MS). Os dados foram submetidos à análise de variância, pelo teste de $F(p>0,05)$ e os tratamentos pela análise de regressão. Não foram observadas interações significativas entre os efeitos simples, entretanto

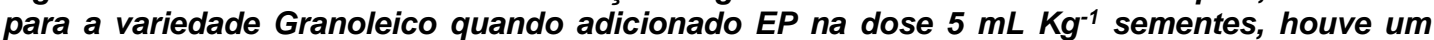
incremento para a porcentagem de emergência, índice de velocidade de emergência e massa seca plântulas ${ }^{-1}$. Já com relação ao comprimento tanto da parte aérea, como raízes $(\mathrm{cm}$ plântulas ${ }^{-1}$ ), maiores valores foram evidenciados para o de $7,5 \mathrm{~mL}$ de EP $\mathrm{Kg}^{-1}$, também para a cultivar Granoleico. Pode-se concluir que extrato pirolenhoso na dose $5 \mathrm{~mL} \mathrm{Kg}^{-1}$, conferiu um melhor desempenho para cultivar Granoleico nos parâmetros de E\%, IVE e CPA, enquanto para a cultivar IAC 503 apenas para o parâmetro de \%E, quando comparado às demais dosagens, para a referida cultura.

Palavras-chave: Arachis hypogaea L; Germinação; Vigor; Vinagre de madeira.

Este é um trabalho de acesso aberto e distribuído sob os Termos da Creative Commons Attribution Attribution-NonCommercial-ShareAlike 4.0 International. 


\title{
Emergence and initial development of peanut seedlings subjected to the use of pyroligneous extract
}

\begin{abstract}
The pyroligneous extract (PE), a product from wood burning, known as pyroligneous acid or wood vinegar, has been studied in several cultures such as corn, beans, rice, capable of exerting effects on germination and seed vigor. Peanut farming is of socioeconomic importance in the regions where it is produced and always seeks to introduce new technologies. In this sense, the aim of this study was to evaluate the influence of the pyroligneous extract on the emergence and initial development of peanut seedlings. The experiment was carried out in a completely randomized design, in a $2 \times 5$ factorial scheme, with two peanut cultivars: IAC 503 and Granoleico; and five doses of EP: T1- $10.0 \mathrm{~mL}$ of deionized water $\mathrm{kg}^{-1}$ of seeds; T2- $2.5 \mathrm{~mL}$ $E P+7.5 \mathrm{~mL}$ deionized water $\mathrm{kg}^{-1}$ seeds; T3- $5.0 \mathrm{~mL} \mathrm{EP}+5.0 \mathrm{~mL}$ deionized water $\mathrm{kg}^{-1}$ seeds; T4-

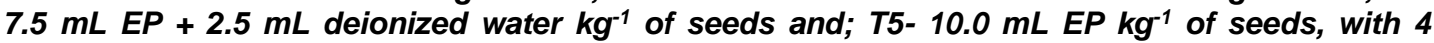
repetitions. Sowing was carried out in $11 \mathrm{dm}^{3}$ polyethylene pots, and the parameters of emergence percentage (E\%), emergence velocity index (EVI), aerial part length (APL), root length $(R L)$ and mass were evaluated. seedling dry matter (DM). Data were subjected to analysis of variance by the $F$ test $(p>0.05)$ and treatments by regression analysis. No significant interactions were observed between the simple effects, however, for the Granoleico variety,

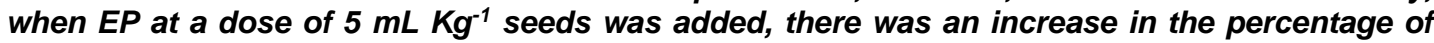
emergence, emergence speed index and seedling dry mass ${ }^{-1}$. As for the length of both shoots and roots (cm seedling ${ }^{-1}$ ), higher values were found for $7.5 \mathrm{~mL}$ of $E P \mathrm{Kg}^{-1}$, also for the Granoleico cultivar. It can be concluded that pyroligneous extract at a dose of $5 \mathrm{~mL} \mathrm{Kg}^{-1}$ provided better performance for cultivar Granoleico in the parameters of E\%, EVI and APL, while for the cultivar IAC 503 only for the parameter of E\%, when compared to the other dosages for that culture.
\end{abstract}

Keywords: Arachis hypogaea L.; Germination; Vigor; Wood vinegar.

\section{Surgimiento y desarrollo înicial de plántulas de mani sometidas al uso de extracto piroleñoso}

\section{RESUMEN}

El extracto piroleñoso (PE), producto de la quema de leña, conocido como ácido piroleñoso o vinagre de madera, ha sido estudiado en diversas culturas como maíz, frijol, arroz, capaz de ejercer efectos sobre la germinación y vigor de la semilla. El cultivo del maní es de importancia socioeconómica en las regiones donde se produce y siempre busca introducir nuevas tecnologías. En este sentido, el objetivo de este estudio fue evaluar la influencia del extracto piroleñoso en la emergencia y desarrollo inicial de plántulas de maní. El experimento se realizó en un diseño completamente al azar, en un esquema factorial $2 \times 5$, con dos cultivares de maní: IAC 503 y Granoleico; y cinco dosis de EP: $\mathrm{T1}-10,0 \mathrm{~mL}$ de agua desionizada $\mathrm{kg}^{-1}$ de semillas; T2- 2,5 mL de EP + 7,5 mL de agua desionizada $\mathrm{kg}^{-1}$ semillas; T3- 5,0 mL EP + 5,0 mL de agua desionizada $\mathrm{kg}^{-1}$ de semillas; $\mathrm{T4}-7,5 \mathrm{~mL}$ de EP $+2,5 \mathrm{~mL}$ de agua desionizada $\mathrm{kg}^{-1}$ de semillas y; T5- 10,0 mL EP $\mathrm{kg}^{-1}$ de semillas, con 4 repeticiones. La siembra se realizó en macetas de polietileno de $11 \mathrm{dm}^{3}$ y se evaluaron los parámetros de porcentaje de emergencia (E\%), índice de velocidad de emergencia (IVE), longitud de la parte aérea (CPA), longitud de la raíz (CPR) y masa. Los datos fueron sometidos a análisis de varianza por la prueba $F$ ( $p>0.05$ ) $y$ tratamientos por análisis de regresión. No se observaron interacciones significativas entre los efectos simples, sin embargo para la variedad Granoleico, cuando se agregó EP a una dosis de $5 \mathrm{~mL} \mathrm{Kg^{-1 }}$ de semillas, hubo un aumento en el porcentaje de emergencia, índice de velocidad de emergencia y masa seca de plántula-1. En cuanto a la longitud tanto de brotes como de raíces (cm plántulas ${ }^{-1}$ ), se encontraron valores mayores para $7,5 \mathrm{~mL}$ de EP $\mathrm{Kg}^{-1}$, también para el cultivar Granoleico. Se puede concluir que el extracto piroleñoso a dosis de $5 \mathrm{~mL} \mathrm{Kg}^{-1}$ brindó mejor desempeño para el cultivar Granoleico en los parámetros de\% E, IVE y CPA, mientras que para el cultivar IAC 503 solo para el parámetro de\% E, en comparación con las otras dosis para ese cultivo.

Palabras clave: Arachis hypogaea L.; Germinación; Fuerza; Vinagre de madera. 


\section{Introdução}

Nos últimos anos, a utilização de produtos obtidos a partir de fontes renováveis e sustentáveis têm tido um expressivo crescimento em seu uso, em especial, na agricultura, com o objetivo de preservar o meio ambiente, bem como assegurar uma boa produção. Muitos desses "novos produtos" consistem em um novo processamento sendo convertidos em subprodutos de valor agregado. Um desses subprodutos é o extrato pirolenhoso (EP), oriundo da queima da madeira para obtenção de carvão vegetal ou madeira torrada.

A recuperação e a sua correta utilização pode minimizar o impacto ambiental causado pelo lançamento dos gases na atmosfera, contribuindo com a redução do aquecimento global (PORTO et al., 2007). O EP é obtido a partir da condensação e recuperação dos gases voláteis gerados na queima da biomassa vegetal, na forma de um líquido de coloração amarela a marrom avermelhada, e também conhecido como ácido pirolenhoso ou vinagre de madeira, sendo composto principalmente de água (80 a 90\% v/v) e uma mistura complexa de milhares de compostos, contendo nesses mais de 200 compostos orgânicos, como ácido acético, álcoois, acetonas, ésteres, fenóis e alguns derivados de lignina, hidrocarbonetos e compostos nitrogenados (GUILLÉN et al., 1995; GUILLÉN; IBARGOITIA, 1996a, 1996b, 1998, 1999; GUILLÉN et al., 2001; ADRIANSZ et al., 2000 - citados por SILVEIRA, 2010).

Silveira (2010) observou que o EP aplicado em solução (10 mL kg-1 de sementes) com diferentes concentrações (0, 25, 50, 75 e 100\%) em sementes de milho, não influenciou a porcentagem de germinação e aumentou, quando na concentração de 25\%, o vigor das plântulas, através dos testes de primeira contagem de germinação (PCG) e do índice de velocidade de emergência (IVE). Van Staden et al. (1995), Jäger et al. (1996), Van Staden et al. (2000) e Van Staden et al. (2006), em seus estudos afirmaram que o EP propiciou efeitos sinérgicos na germinação e no crescimento radicular de plântulas de alface, crisântemo, tomate, quiabo, feijão e milho. 
Diante do exposto, torna-se importante a utilização de substâncias que contenham compostos capazes de proporcionar efeitos na germinação e vigor das sementes, possibilitando incrementos na produção, resultando em aumentos significativos de produção e produtividade. Nesse sentido, o presente estudo objetivou avaliar a influência do extrato pirolenhoso (EP) na emergência e desenvolvimento inicial de plântulas de amendoim.

\section{Material e métodos}

O experimento foi conduzido sob condições de campo, em uma propriedade, no município de Jaboticabal-SP, nos meses de outubro a novembro de 2020. O clima da região é classificado como Aw segundo a classificação climática de Köppen e Geiger, com temperatura média de 21,7 ${ }^{\circ} \mathrm{C}$ e pluviosidade média anual de $1340 \mathrm{~mm}$. Foram utilizadas sementes de amendoim cultivares IAC 503 e Granoleico, com ciclos ao redor de 130 dias, da safra 2019/2020, tratadas com os ingredientes ativos (i.a.) tiametoxam e fipronil, fertilizante líquido Basfoliar ${ }^{\circledR}$ CoMol Top SL.

Em adição ao tratamento de sementes, fora utilizado o Extrato Pirolenhoso (EP). Para tal, utilizou-se vasos de $11 \mathrm{dm}^{3}$, em ambiente aberto, sob tablado de madeira, dispostos em um delineamento inteiramente casualizado, em esquema fatorial $2 \times 5$, sendo as duas cultivares de amendoim e cinco doses de EP: T1- 10,0 mL de água deionizada $\mathrm{kg}^{-1}$ de sementes; T2- 2,5 mL EP + 7,5 mL de água deionizada $\mathrm{kg}^{-1}$ de sementes; T3- 5,0 mL EP + 5,0 mL de água deionizada $\mathrm{kg}^{-1}$ de sementes; T4- 7,5 mL $\mathrm{EP}+2,5 \mathrm{~mL}$ de água deionizada $\mathrm{kg}^{-1}$ de sementes; T5- 10,0 mL EP kg-1 de sementes. Foram utilizadas quatro repetições, contemplando 40 unidades experimentais. As variáveis analisadas foram dadas pela porcentagem de emergência de plântulas (\%E), conduzido em areia lavada, previamente peneirada. As sementes foram semeadas a uma profundidade de $2,5 \mathrm{~cm}$, contendo 25 sementes por vaso de cada cultivar, com seus respectivos tratamentos, sendo estes mantidos pelo período de 10 dias, seguindo observações semelhantes às das Regras para Análise de Sementes (BRASIL, 2009), caracterizando uma metodologia controlada e embasada no desenvolvimento inicial das plântulas de amendoim. No final do $10^{\circ}$ dia, 
foram contabilizadas as plântulas normais emergidas, dadas em porcentagem.

Em conjunto com o teste de porcentagem de emergência (E\%), foi realizado o índice de velocidade de emergência de plântulas (IVE), anotando-se o número de plântulas que apresentaram as folhas visíveis. Ao final do teste foi calculado, segundo os dados diários do número de plântulas normais, o IVE empregando-se a fórmula proposta por MAGUIRE (1962). Na sequência e em conjunto com o teste de porcentagem de emergência, foram realizados os testes de comprimento de plântulas (CP), medindo-se a parte aérea (CPA) e sistema radicular (CPR), considerando 10 plântulas normais, por unidade experimental, de cada repetição.

A CPA fora medida do colo da plântula até o seu ápice foliar, enquanto a CPR do colo até o final do sistema radicular aparente, com o auxílio de uma régua milimetrada, expresso em $\mathrm{cm}$ plântula ${ }^{-1}$. Ainda, foi realizado o teste de massa de matéria seca de plântulas (MSP), mensurando os valores da parte aérea (MSPA) e massa seca de raízes (MSR), sendo as plântulas advindas do teste de comprimento de plântulas. As plântulas foram acondicionadas em sacos de papel kraft, sendo estas secas ao sol pelo período de 96 horas e, posteriormente, levadas em estufa para secagem a $65^{\circ} \mathrm{C}$, por 72 horas, até atingirem peso constante, com a pesagem do material sendo feita em balança analítica de precisão $0,01 \mathrm{~g}$, e os valores dados em g plântula ${ }^{-1}$.

Os dados foram submetidos à análise de variância pelo teste $\mathrm{F}$ $(p<0,05)$. Utilizou-se análise de regressão para o efeito de doses e teste de Tukey, a $5 \%$ de probabilidade, para o efeito nas cultivares, por meio do software estatístico SISVAR (FERREIRA, 2019).

\section{Resultados e discussão}

Pelo teste $F$ não foram verificadas interações significativas $(p>0,05)$ entre fator simples de cultivares e fator EP para nenhum dos parâmetros estudados. Para as cultivares foi verificada diferenças significativas para todos os parâmetros estudados, com exceção da MSP. Para as doses de EP 
foram verificadas diferenças significativas apenas para os parâmetros de E\% e CPA. A cultivar Granoleico apresentou os maiores percentuais de emergência quando comparada com a cultivar IAC 503. Tanto para a cultivar IAC 503 quanto para a Granoleico a dose de 5,0 mL de EP kg-1 de sementes foi a que proporcionou maiores valor para $\mathrm{E} \%$, com os menores valores de E\% obtidos na dose de 10,0 mL de EP kg-1 de sementes para a IAC $503 \mathrm{e}$ do tratamento sem EP para a Granoleico (Tabela 1).

Tabela 1. Desdobramento do uso de doses do Extrato Pirolenhoso (EP) no tratamento de sementes e de duas cultivares de amendoim (IAC 503; Granoleico). Jaboticabal-SP, 2020.

\begin{tabular}{|c|c|c|c|c|c|}
\hline DOSES $^{(1)}$ & 0,0 & 2,5 & 5,0 & 7,5 & 10,0 \\
\hline $\mathrm{E} \%$ & \multicolumn{5}{|c|}{ Porcentagem de emergência (\%) } \\
\hline IAC 503 & $26,75 a b B^{2}$ & $26,00 \mathrm{abB}$ & $32,00 \mathrm{aB}$ & $27,00 \mathrm{abB}$ & $19,50 \mathrm{bB}$ \\
\hline Granoleico & $42,50 \mathrm{cA}$ & $45,00 \mathrm{bcA}$ & $60,00 \mathrm{aA}$ & $55,00 \mathrm{abA}$ & $49,00 a b c A$ \\
\hline IVE & \multicolumn{5}{|c|}{ Índice de Velocidade de Emergência } \\
\hline IAC 503 & $2,72 \mathrm{~B}$ & $1,79 \mathrm{~B}$ & $2,85 \mathrm{~B}$ & $1,99 \mathrm{~B}$ & $1,74 \mathrm{~B}$ \\
\hline Granoleico & $4,32 \mathrm{bA}$ & $5,30 \mathrm{abA}$ & 6,43 aA & $6,21 \mathrm{aA}$ & $5,14 a b A$ \\
\hline CPA & \multicolumn{5}{|c|}{ Comprimento da parte aérea (cm plântula-1 $)$} \\
\hline IAC 503 & $2,10 \mathrm{~B}$ & $2,36 \mathrm{~B}$ & $2,52 \mathrm{~B}$ & $2,22 \mathrm{~B}$ & $2,12 \mathrm{~B}$ \\
\hline Granoleico & $4,35 a b A$ & $4,70 \mathrm{abA}$ & $4,75 \mathrm{aA}$ & 5,09 aA & $3,55 \mathrm{bA}$ \\
\hline CPR & \multicolumn{5}{|c|}{ Comprimento de raízes (cm plântula ${ }^{-1}$ ) } \\
\hline IAC 503 & $12,10 \mathrm{~B}$ & $12,97 \mathrm{~B}$ & $14,30 \mathrm{~A}$ & $13,19 \mathrm{~B}$ & $11,83 \mathrm{~B}$ \\
\hline Granoleico & $15,77 \mathrm{~A}$ & $16,52 \mathrm{~A}$ & $14,40 \mathrm{~A}$ & $17,72 \mathrm{~A}$ & $15,55 \mathrm{~A}$ \\
\hline MSP & \multicolumn{5}{|c|}{ Massa seca de plântulas (g plântula-1) } \\
\hline IAC 503 & $8,30 \mathrm{~A}$ & $9,65 \mathrm{~A}$ & $9,37 \mathrm{~B}$ & $9,72 \mathrm{~A}$ & $9,53 \mathrm{~A}$ \\
\hline Granoleico & $9,13 \mathrm{~A}$ & $9,76 \mathrm{~A}$ & $11,30 \mathrm{~A}$ & $10,20 \mathrm{~A}$ & $9,77 \mathrm{~A}$ \\
\hline $\begin{array}{l}\text { DOSES - tr } \\
\text { lédias seguic }\end{array}$ & ntos $(e m$ & de EP $\mathrm{kg}^{-1}$ & mentes); & extrato pi & $\begin{array}{l}\text { nhoso; } \\
\text { una, não }\end{array}$ \\
\hline
\end{tabular}

Chumpooam et al. (2012) citados por José et al. (2016), ao avaliarem altas doses de EP verificaram que ocorreu a redução da porcentagem de germinação, que em comparação às doses mais baixas, resultado similar ao comportamento apresentado no presente estudo, em que a $\mathrm{E} \%$ apresentou um modelo quadrático de regressão quanto aso uso das doses de EP, estimando para a cultivar Granoleico E\% máxima de $56,09 \%$, na dose de $6,09 \mathrm{~mL}$ de EP kg-1 de sementes, enquanto que para a cultivar IAC 503, a máxima E\% foi de $29,43 \%$, na dose de $3,93 \mathrm{~mL}$ de $\mathrm{EP} \mathrm{kg}^{-}$ ${ }^{1}$ de sementes (Figura 1). 


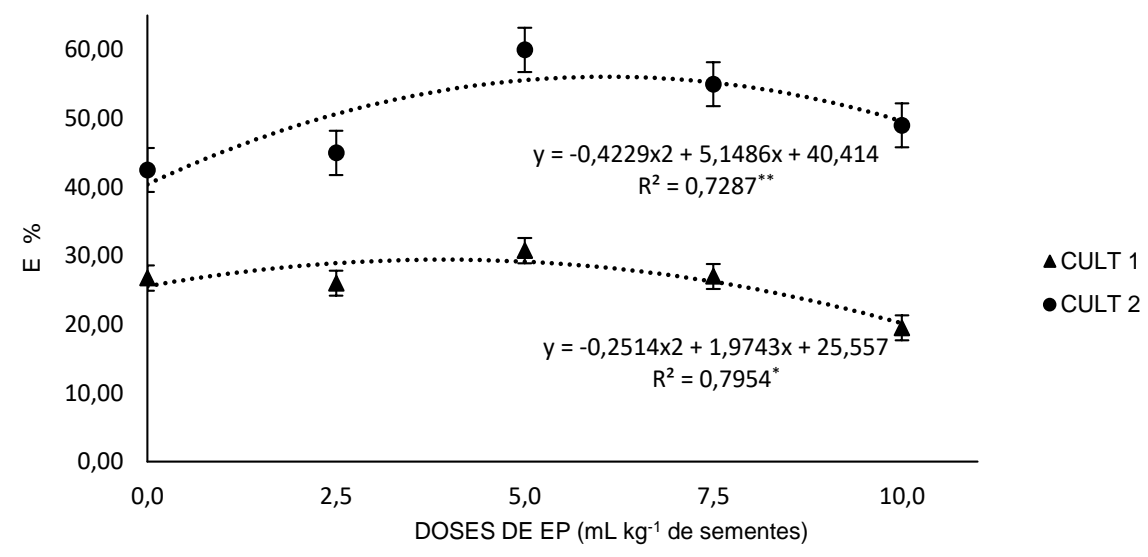

Figura 1. Porcentagem de Emergência de Plântulas (E\%) de duas cultivares de amendoim (CULT 1 - IAC 503 e CULT 2 - Granoleico) após a aplicação de extrato pirolenhoso via sementes. Jaboticabal, 2020.

Van Staden et al. (2000) com a utilização do extrato pirolenhoso, diluído em 1:50 (v/v), relataram a existência de possível efeito sinérgico durante 0 processo de embebição da semente, propiciando 0 desenvolvimento embrionário acelerado, antes mesmo do surgimento da radícula. Porém as concentrações mais elevadas de EP, com 50, 75 e 100\% aplicadas em solução de $10 \mathrm{~mL} \mathrm{~kg}^{-1}$ de sementes, na presente pesquisa, apresentarem valores médios similares aos da testemunha.

$\mathrm{Na}$ tabela 1, considerando o IVE, os maiores valores foram obtidos para a cultivar Granoleico quando comparada com a IAC 503. Não foram verificadas diferenças significativas entre os tratamentos para cultivar IAC 503, o que indica que o IVE não foi afetado pelos tratamentos aplicados às sementes. Entretanto, para a cultivar Granoleico, os tratamentos diferenciaram significativamente entre si, indicando influência do EP sobre o IVE das sementes, com os maiores valores médios observados para as doses de 5,0 e 7,5 mL de EP kg-1 de sementes, enquanto os menores foram obtidos para dose de 10,0 $\mathrm{mL}$ de EP $\mathrm{kg}^{-1}$ de sementes. Silveira (2010) avaliando plântulas de milho, verificou que IVE na concentração de $25 \%$ de EP proporcionou maior velocidade de germinação às sementes de milho, quando comparada com a solução contendo $0 \%$ de $\operatorname{EP}(p<0,05)$. 
Foram verificadas diferenças significativas entre as cultivares em CPA, com a IAC 503 apresentando os menores valores médios de CPA e a Granoleico, os maiores. Não houve diferenças significativas entre os tratamentos aplicados às sementes de IAC 503, enquanto para a cultivar Granoleico apresentou diferenças significativas entre os tratamentos, sendo que os maiores valores médios para o desenvolvimento da parte aérea foram observados para as doses de 5,0 e 7,5 mL de EP kg-1 de sementes (Tabela 1).

Para a CPR de plântulas de amendoim, a cultivar IAC 503 apresentou valores médios significativamente menores, estatisticamente, quando em comparação ao Granoleico. Não foram verificadas diferenças significativas entre os tratamentos com EP, para ambas cultivares, o que demonstra que esses tratamentos não exerceram efeitos significativos, porém, apresentaram valores médios maiores do que a testemunha, para todas as doses, quanto ao desenvolvimento radicular do amendoim (Tabela 1), nas condições estudadas.

Para a MSP, não foram observadas diferenças significativas entre as doses de EP utilizadas e a testemunha, porém todos os tratamentos apresentaram maiores valores médios para o acúmulo de MSP, nas plântulas de amendoim, para as duas cultivares testadas. E, entre as cultivares, a Granoleico apresentou maiores valores médios de MSP (Tabela 1). Ainda, foi observado aumento da MSP entre as doses de 5,0 e 7,5 mL de EP kg ${ }^{-1}$ de sementes, ajustando-se ao modelo quadrático de regressão, para as doses testadas (Figura 2).

Os efeitos positivos do EP para a cultivar IAC 503 estão entre as doses de 3,93 a 6,64 $\mathrm{mL}^{\text {de EP kg-1 }}{ }^{-1}$ de sementes, enquanto para a cultivar Granoleico se encontram entre 4,39 a 6,09 $\mathrm{mL}^{\text {de EP kg-1 }}$ de sementes, proporcionando maior desenvolvimento inicial das plântulas de amendoim, para as duas cultivares estudadas e, possibilitando o uso do produto na cultura, além de sugerir a execução de novos trabalhos científicos, para sua aplicação no tratamento de sementes. 


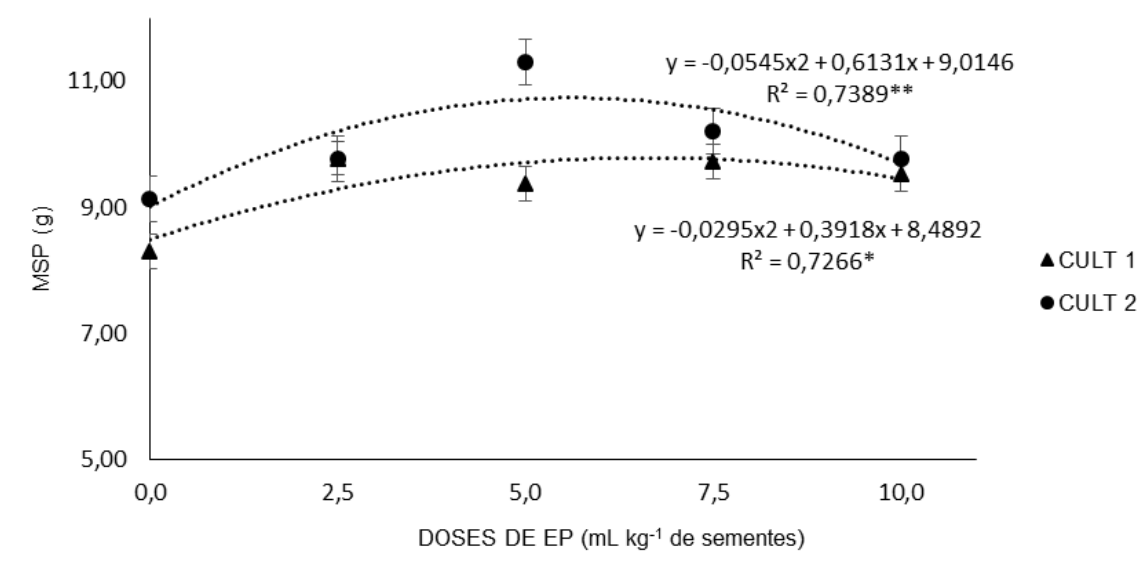

Figura 2 Massa seca de plântulas (MSP) de duas cultivares de amendoim (CULT 1 - IAC 503 e CULT 2 - Granoleico) após a aplicação de extrato pirolenhoso via sementes. Jaboticabal, 2020.

\section{Conclusões}

A dose de EP de $5,0 \mathrm{~mL} \mathrm{~kg}^{-1}$ de sementes conferiu melhor desempenho para cultivar Granoleico nos parâmetros de E\%, IVE e CPA, enquanto que para a cultivar IAC 503 apenas para o parâmetro de E\%, quando comparado às demais dosagens, para a referida cultura, no tratamento de sementes.

\section{Agradecimentos}

À empresa ECOGRILL EXTRATO PIROLENHOSO EIRELI, no município de Mauá da Serra - PR, pelo apoio na disponibilidade do produto e custeio de projetos de pesquisa na referida área de estudo. 


\section{Referências}

BRASIL. Ministério da Agricultura, Pecuária e Abastecimento. Secretaria de Defesa Agropecuária. Regras para análise de sementes. Brasília: Mapa/ASC, 2009. 399p.

FERNANDES, $L$ et al. Extrato pirolenhoso no desenvolvimento de Catasetum osculatum Lacerda \& P. Castro. In: SEMINÁRIO DE BIODIVERSIDADE E AGROECOSSISTEMAS AMAZÔNICOS, 3., 2015, Alta Floresta. Anais [...] . Alta Floresta: Universidade do Estado de Mato Grosso, 2015. p. 518-522.

FERREIRA, D. F. SISVAR: a computer analysis system to fixed effects split plot type designs. Revista Brasileira de Biometria, [S.I.], v. 37, n. 4, p. 529535, dec. 2019.

JÄGER, A.K.; STRYDOM, A; VAN STADEN, J The effect of ethylene, octanoic acid and a plant-derived smoke extract on the germination of lightsensitive lettuce seeds. Plant Growth Regulation, Pietermaritzburg, v. 19, p. 197-201, 1996.

JOSÉ, A. C. et al. Efeito do extrato pirolenhoso sobre a germinação de espécies do Cerrado brasileiro. Caderno de Ciências Agrárias, v. 8, n. 1, p. 62-69, 2016.

MAGUIRE, J. D. Speed of germination-aid in selection and evaluation for seedlig emergence and vigor. Crop Science, v. 2, n.1, p.176-177, 1962.

PORTO, P. R.; SAKITA, A. E. N.; NAKAOKA SAKITA, M. Efeito da aplicação do extrato pirolenhoso na germinação e no desenvolvimento de mudas de Pinus elliottii var. elliottii. Instituto Florestal Série Registros, São Paulo, n. 31, p. 15-19, 2007.

SILVEIRA, C. M. Influência do extrato pirolenhoso no desenvolvimento e crescimento de plantas de milho. 2010. 93 f. Tese (Doutorado) - Curso de Agronomia, Universidade Estadual Paulista, Jaboticabal, 2010.

VAN STADEN, J.; BROWN, N. A. C.; JÄGER, A. K.; JOHNSON, T. A. smoke as a germination cue. Plant Species Biology, Sapporo, v. 15, n. 2, p. 167$178,2000$.

VAN STADEN, J.; JIIGER, A.K.;STRYDOM, A. Interaction between a plantderived smoke extract, light and phytohormones on the germination of lightsensitive lettuce seeds. Plant Growth Regulation, Pietermaritzburg, v. 17, p. 213-218, 1995.

VAN STADEN, J.; SPARG, S. G.; KULKARNI, M. G.; LIGHT, M. E. Postgermination effects of the smoke-derived compound 3-methyl-2Hfuro[2,3c]pyran-2-one, and its potential as a preconditioning agent. Field Crops Research, Amsterdam, v. 98, p. 98-105, 2006. 